\title{
From frailty to hope: the Yin and Yang of nephrology
}

\section{Giorgina Barbara Piccoli ${ }^{1}$}

Published online: 1 October 2021

(c) Italian Society of Nephrology 2021
While choosing the papers to include in the present issue, I realised that two aspects had recently attracted the attention of our contributors, and that they represent the Yin and Yang of our discipline: the frailty of our patients with advanced CKD, on dialysis or after kidney transplantation, and the fight for life in obstetric nephrology.

The papers included in this issue relate to two major themes: vascular diseases and fragility, and pregnancy and women's health. Miscellanea of themes closes the issue in an attempt to convey what clinical activity involves. Unlike previous issues, the current one is organised in three sections: frailty and vascular disease, pregnancy and related issues, and a collection of papers on different topics. At the end of each section there are teaching cases, lessons and letters for readers to enjoy.

The opening paper is a pragmatic review whose evocative title, "The Fragile World of Haemodialysis Patients During the COVID-19 Pandemic", examines both traditional methods of caring for a greying population and the new concerns that arose during the pandemic. The papers that follow explore old and new problems, from the management of hyperparathyroidism in pre-dialysis patients, to the role of hypertension in chronic kidney disease, dialysis and after transplantation, and the importance of lipid lowering treatment, as well as their controversial use. Oral anticoagulant therapy is discussed in a commentary, while two epidemiological studies remind us of the importance of the imperative of primum non nocere, reporting on the risk of contrast induced acute kidney injury. Vascular health, the Achilles heel of our patients, is explored from different perspectives: the elusive diagnosis of kidney infarction, compromised vascular health at dialysis start, witnessed by erectile dysfunction in incident patients, often neglected by physicians but central to patients' quality of life.

Giorgina Barbara Piccoli

gbpiccoli@yahoo.it

1 Nephrologie, Centre Hospitalier Le Mans, 7200 Le Mans, France
The challenges of calcyphylaxis, one of the most feared vascular complications in dialysis patients, quantified in a nationwide study, are proposed in two interesting cases. Tailoring treatment for elderly patients and for individuals with a long dialysis vintage encourages us to reflect on the balance between standardization and personalization.

Perhaps there is nothing new under the sun, and yet much has been progress has been made (Fig. 1). A historic commentary reviews the epidemiology of gout, through the history of the Roman popes.

From the past to the future, a rich series of studies explore the still mysterious relationship between the kidney and pregnancy: an expert team shares data on the rare and dreaded pregnancy-induced atypical haemolytic uremic syndrome, recalled also in a case-based lesson. Nothing is trivial in pregnancy, and a systematic review finds that even kidney stones are related to adverse pregnancy outcomes; a nationwide study on IgA nephropathy, the most common glomerulonephritis in pregnancy, underlines its heterogeneity. At the opposite end of the severity spectrum, is a Japanese survey of pregnancy in patients on haemodialysis that we are happy to publish.

The relationship between pregnancy and kidney disease is bidirectional: pregnancy-induced AKI is still a challenge all over the world, while new data from the well-known Tehran lipid study cohort confirm the importance of preterm delivery as a marker of future kidney health.

Identifying the problem is just the first step, however; much needs to be done to support mothers' health: it is time that our scientific societies, as well as our stakeholders, acknowledge the importance of post natal assessment, at least of kidney function and proteinuria, to try to transform the difficult experience of preeclampsia into early diagnosis and prevention of CKD.

After miscellaneous contributions on topics ranging from peritoneal catheter placement to high volume haemodiafiltration and the long-term risk of childhood diseases, the journal closes with the hope that the frequent lack of precise CKD diagnosis can be remedied through a wider use of genetic testing. 


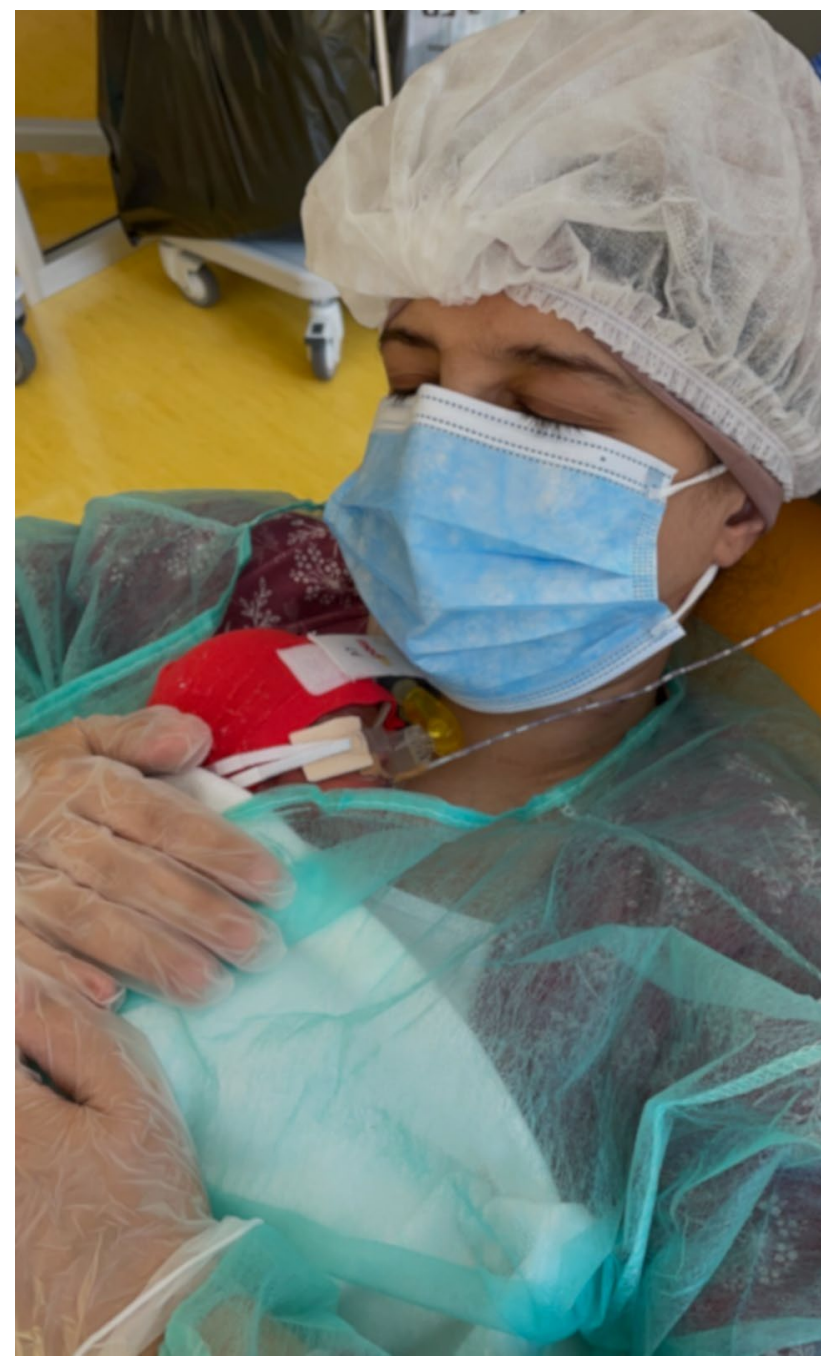

Fig. 1 Mother and daughter. After 6 years of haemodialysis, this young woman delivered a premature baby girl, now developing well. The mother's pride and the daughter's fragility remind us of the challenges we still have to face in dialysis and in obstetric nephrology. (Photo taken by Linda Gammaro, a long-time member of the Kidney and Pregnancy Study Group of the Italian Society of Nephrology. Courtesy of LG, used with the patient's permission)
As editors we were challenged during the COVID-pandemic by almost double the number of submissions we normally receive. Many of them were innovative, exploring new aspects of nephrology and unmet needs of renal care. JN is not alone; the number of submissions to several nephrology journals increased, demonstrating our resilience and capacity for reinvesting our time in research. The quality and variety of these contributions, and the commitment of the editorial team are proof of this reinvestment. For the sake of our patients, I hope that it will last beyond the much-awaited end of the pandemic.

Acknowledgements To Susan Finnel for her careful language correction.

\section{Declarations}

Conflict of interest No conflict of interest or financial sources declared.

Ethical approval No financial support and no ethic committee approval was needed for this editorial.

Publisher's Note Springer Nature remains neutral with regard to jurisdictional claims in published maps and institutional affiliations. 